\title{
Providers' constructions of pregnant and early parenting women who use substances
}

\section{Cecilia Benoit $^{1}$, Camille Stengel ${ }^{2}$, Lenora Marcellus ${ }^{3}$, Helga Hallgrimsdottir ${ }^{4}$, John Anderson' ${ }^{6}$, Karen MacKinnon $^{3}$, Rachel Phillips ${ }^{6}$, Pilar Zazueta ${ }^{5}$ and Sinead Charbonneau'}

${ }^{1}$ Centre for Addictions Research of British Columbia and Department of Sociology, University of Victoria, Canada

${ }^{2}$ School of Social Policy, Sociology and Social Research, University of Kent, UK

${ }^{3}$ School of Nursing, University of Victoria, Canada

${ }^{4}$ Department of Sociology, University of Victoria, Canada

${ }^{5}$ Division of Medicine, University of Victoria, Canada

${ }^{6}$ Centre for Addictions Research of British Columbia, University of Victoria, Canada

Abstract The research literature indicates that problematic substance use as a form of health behaviour is poorly understood, being sometimes viewed as deviance, at other times as a disease, and most often as a combination of these states. The use of substances by women who are pregnant or new parents is often conceptualised within an individualised framework. Yet drinking alcohol and using other drugs during pregnancy and early parenthood cuts across social divisions and is shaped by sociostructural contexts including health care. There is a growing body of literature that critically examines public health interventions that are aimed at implementing harm reduction and health promotion techniques in service delivery to help pregnant and early parenting women who are identified as problem substance users. We examine qualitative data from representatives of a recent harm reduction intervention, focusing, in particular, on providers' individual conceptualisations of the problematic behaviour. Our results show that most study participants regard any substance use during pregnancy, birth and the postpartum period as fundamentally unacceptable. This framing of problematic substance use is accomplished via gendered responsibilisation of women as foetal incubators and primary caregivers of infants. We discuss our results in light of the current literature and suggest policy implications.

Keywords: health behaviour, problematic substance use, pregnant and early parenting women, harm reduction

\section{Introduction}

Use of substances by women who are pregnant or new parents is often conceptualised within an individualised framework. It is commonplace to employ the language of substance abuse when referring to pregnant women who use drugs and other substances and who are targeted 
for intervention (Campbell and Alexander 2006, Claus et al. 2007, Lefebvre et al. 2010). While it seems to be a minor problem, this choice of language suggests that pregnant women who use substances are breaching their ethical responsibilities to themselves and the foetus: by 'choosing to abuse' substances and allowing themselves to become addicted they have become 'pregnant addicts' (Young 1994). Rutman et al. (2000) state that this moralising language tends to 'imply some amount of judgment toward the woman and encourage an avenue of thinking that separates her from the context of her life' (p.v).

The belief that individuals are chiefly responsible for their health behaviour fundamentally shapes theories of health, disease and addiction to alcohol and other substances (Room 1983, Reinarman 2005, Schneider 1978). This notion also informs harm reduction approaches that aim to empower individuals with the tools and services to change substance use patterns, learn to consume substances in a safer manner, and engage in other forms of behaviour to reduce drug-related harm (Lenton and Single 1998).

Results from research studies have shown that drinking alcohol and using other drugs during pregnancy and early parenthood cuts across social divisions; yet the societal disapproval and accompanying stigma of substance use tends to be disproportionately attached to women of disadvantaged backgrounds (Campbell and Ettorre 2011, Lester et al. 2004). Researchers used urine samples to test the substance use of pregnant women in a Florida county and found that although the rates of substance use were fairly equal between private and public clinics and between Black and White women, Black women were ten times more likely than White women to be reported to health authorities based on their practitioners' risk assessments, and poor women were also reported at a much higher frequency than wealthy women (Chasnoff et al. 1990). Other studies have documented unequal testing for substance use comparing White populations with ethnic minorities (Kerker et al. 2006, Lloyd 2010). Finally, although prescription drug use and misuse during pregnancy is on the rise in many countries, the more punitive focus from health and social services authorities continues to be on illicit substance use (such as marijuana, cocaine, heroin and crystal methamphetamine), again often bringing attention to women from more disadvantaged backgrounds (Campbell and Ettorre 2011).

Pregnant and early parenting women who are likely to be identified as problem substance users also experience various forms of social inequity and marginalisation that often precede substance use, including inadequate material resources, unstable or deficient housing and low education (Bailey et al. 2012, Greaves and Poole 2008, Schempf and Strobino 2008). In the USA many women in this situation also lack health insurance (Roberts and Pies 2011). These factors have been identified as necessary considerations for contextualising drug use during the reproductive period, but also as evidence of the way that surveillance of maternal substance use intersects with class and racial discrimination (Salmon 2011). Furthermore, the stigma associated with maternal substance use engenders a host of social, material and psychological marginalisations that have adverse consequences for both the mother and her child, including their avoidance of services even when they are available for fear of being harshly judged (Poole and Isaac 2001).

While the recent growth of targeted programmes aim to minimise the risk of health and social problems for disadvantaged mothers, many of these programmes and associated policies are oriented towards identifying individual risk and tend to employ social surveillance in the form of risk assessments of infants by the state, rather than focusing on the empowerment and inclusion of women of disadvantaged backgrounds who lack access to key social determinants of health (Murphy 2000, Zadoroznyj 2006).

Unlike these individualised treatment programmes, community-based programmes are inclusive, participant-centred, harm reduction interventions that focus on addressing both the social and health dimensions of substance use during pregnancy and early parenthood. Since the 1990s a handful of such harm reduction integrated programmes have emerged across Canada, including 
Breaking the Cycle and New Choices in Ontario, the Maxxine Wright Place Project in the Fraser Valley region of British Columbia (BC) and Sheway in Vancouver, BC. HerWay Home (HWH), an abbreviation for 'housing first, empowerment, respect, women, acceptance, your choice, health, opportunity, mother and equality', is situated in Victoria, BC, and builds on these earlier successful initiatives. The programme was officially launched in 2013 and is designed to be a cross-sectorial, community-based, culturally safe service site for women, infants and families who are experiencing barriers to health care due to intersecting marginalisation. The HWH core programme services include basic needs support (for example, nutrition and child-minding), primary health and perinatal services, counselling for substance use, trauma and mental health issues and support in accessing and maintaining housing. HWH is the result of efforts by an interdisciplinary and cross-sector network of local health and social care professionals, outreach agencies, members of charitable organisations and government agencies, and an advisory group of women who identify as representatives of the HWH service population. The HWH initiative is informed by a harm reduction philosophy and a social determinants of health framework that together recognise the importance of providing services to help reduce the harm associated with substance use while providing access to crucial resources such as safe and secure housing, income assistance, social support, food security and educational and employment opportunities.

There is a growing body of academic literature that critically examines recent public health interventions, such as HWH, that are aimed at implementing harm reduction and health promotion techniques in service delivery. This literature situates public health discourse within the context of neoliberalisation, welfare state reform and the social origins of health inequities, highlighting the ways in which new public health discourses advance individual solutions to problems alongside social understandings of health, with adverse and contradictory consequences for disadvantaged groups (Campbell and Ettorre 2011, Cockerham 2005, Greaves and Poole 2008, Link and Phelan 2006, Navarro 2009, Zadoroznyj 2006). Drawing on this literature, we examine qualitative data from representatives of the HWH network of health and social care providers and programme funders. We focus, in particular, on these providers' conceptualisations of problematic substance by women during pregnancy and early parenting. First, we briefly describe our study and methodology.

\section{Sample and methods}

The data presented in this article were gathered as part of a mixed-method study entitled Interventions to Promote Health and Health Equity for Pregnant and Early Parenting Women Facing Substance Use and Other Challenges, which was funded by the Canadian Institute of Health Research and received ethical review from the Human Research Ethics Board at the University of Victoria.

The study aimed to shed light on: (i) the factors that promote open communication and the full participation of all team members of the HWH so that continuity of primary maternity care is established and sustained over time and the clients are treated with respect and dignity by providers; and (ii) the factors that enhance client access to other health and social services that are key in harm reduction and health promotion strategies for women affected by substance use during pregnancy and early parenting. Semi-structured interviews were initially conducted with the core team of health and social care providers connected with the HWH and key local community and provincial governmental stakeholders, and the study was eventually expanded to include interviews with a more diverse sample of providers who had a role in serving pregnant women and persons affected by substance use, but with a more limited knowledge of HWH. Our qualitative results, presented below, are based on interviews conducted with 56 
persons. More specifically, the analysis focuses on providers' responses to the question: 'How do you define problematic substance use among pregnant and early parenting women?'

\section{Findings}

Our findings reveal the most popular conceptions of problematic maternal substance use identified in the data. It was not uncommon for participants to draw on more than one of the broad conceptualisations noted below when seeking to explain problematic substance use. It was not apparent that differences in the emphases that providers placed on certain perceptual frameworks could be linked to their professional background. One exception might be that providers who worked in outreach and other community-based services for street-based populations were more likely draw on the language of harm reduction in their explanations. Many participants who espoused a harm reduction point of view were reluctant to embrace it fully: a response with both semiotic and discursive features that characterise the ethical complexity of foetal and maternal rights for advocates of individualistic harm reduction models.

\section{The difficulty of defining the problem}

While many of the participants work in the context of pregnancy, parenting and substance use in their everyday action as service providers, very few had a ready definition of problematic substance use and, indeed, struggled momentarily with the question. This moment of discomfort in identifying suitable language is likely to be a reflection of the social, legal and moral ambiguity within which maternal substance use behaviour occurs. Thus, the issue of problematic substance use among pregnant and early parenting women was not readily articulable, as illustrated in this participant's response: '[Silence] Oh that's really interesting, I never sort of thought about actually defining it'. Similarly, another participant responded with the answer: 'That's so funny; I've never really sort of considered that, that question'. One participant noted that she does not need to define the concept for her practice:

[I]t's not something I've really thought about defining. I mean, take away problematic and I'm comfortable with ... just working with people who use substances. I don't know whether I'd define problematic substance use in my practice. (Rebecca ${ }^{1}$ )

Two participants stated explicitly that their professional definitions are different from their personal definitions:

[J]ust, whatever my personal thoughts or fears are ... I know that in my role in my job, it's not up to me to be deciding like what's right or wrong for them. (Sarah)

[L]ike, I had a different idea for myself when I was pregnant ... so I guess it kind of varies, like professionally, that's my professional answer. (Emily)

In the case of the latter respondent, she was clearly struggling with her own experience of pregnancy and her professional view of substance use among pregnant clients. Drawing distinctions between and discrete boundaries around the ideologies employed in private and public realms is difficult. However, we report these examples here because they are symptomatic of a more widespread tension among participants to articulate a moral-ethical hierarchy that guides their definitions of problematic maternal substance use and their related practices as service providers. The attempt to separate personal from professional practices is indicative of the degree to 
which maternal substance use can be considered in moral terms over other acts of substance use. Since the views of some of the providers we spoke to reflected popular notions of maternal purity and the sanctity of the foetus, an artificial elision between personal and professional practices occurs, allowing providers to maintain their private ethic and image of appropriate maternal behaviour while still striving to provide care in a non-judgmental way. Whether this division could actually be maintained in practice is unclear, but the need to identify these distinctions indicates that some provider's perceptions are deeply entrenched in moral judgements.

The most common perceptual frameworks that participants drew upon to define problematic substance use focused on terms such as safety, risk and ability to care and the notion that the individual woman is the arbiter of what constitutes problematic substance use in her own life. Some participants acknowledged and foregrounded the ways in which perceptions of problematic substance use are generated in the broader representational hierarchy wherein bodies bound to certain spaces and classes are read and labelled as problematic.

\section{Safety, risk and ability to care}

Just over half the participants focused on problematic substance use as a loss of the ability to care. The ability to care was referenced in relation to keeping oneself safe, securing material resources and, most importantly, the responsibility of the woman to optimise the health of her foetus and infant. Quotes from three different participants are provided as examples:

[I]t might not be so problematic [for a woman]. She might be coping. But it might be problematic for her child: either the physical growth of her baby during pregnancy or her ability to parent that child. (Monica)

[I]f they can say 'I'm gonna go and party, and mom's looking after the baby'... if it's not consuming their life and that's, that's what they do and they go out to the bar and have some drinks I think that's fine within, you know, reason, but ... if the, you know, baby is in the crib and friends come over and, they party or they're, you know, abusing drugs then how can they safely parent their child? (Christine)

You could be middle to upper class and be a regular heroin user but you can afford to pay for a nanny, so therefore someone's taking care of your children. But then, you can argue that the connection between parent and child is harmed. So, for me, the focus would be on the children. (Marlon)

The final two passages also portray the way that maternal responsibility can be offset by the availability of another woman to care; problematic substance use is thus, in part, defined by a lack of substitute maternal care.

While some respondents highlighted women's safety as a dimension of problematic substance use, many of these instances linked women's safety and wellness to foetal health and the care for children, thus positioning women's health as a means to the end of the health of the infant or child. As one participant put it: "when ... substances are interfering with her ability to have a level of stability, and safety for her and either ... for her most importantly, and then for her children'. Another participant similarly commented on the perceived potential of a conflict between women's bodily autonomy and foetal and infant health:

And it's a balancing act, right? We want to be very woman-focused, and I absolutely approach my work from that mind frame, but there's still a baby involved ... I'm very 
pro-choice but ... when she said 'I'm choosing to keep this pregnancy', then it's a baby. It's no longer a foetus. She's made the decision already that it's a baby. (Kate)

While it is certainly true that engaging in practice or discourse about maternal substance use (or, for that matter, the actual practice of mothering) is a balancing act of rights, responsibilities and needs, this participant's narrative posits a morally construed conflict between the mother and the foetus that is not justified by the Canadian legal context (Poole and Isaac 2001). By using the term 'baby', this participant is making an ontological leap with significant normative implications. In deconstructing this participant's discourse, the intention is not to sublimate the experience of providing care for pregnant women who are likely themselves to relate to their foetuses as babies. Faced with this normalising practice on a day-to-day basis, practitioners' dialectical relation to the pregnant woman and her foetus affects language that is contextually important (to validate pregnant women's experiences) but may also have unintended social consequences.

In sum, one can extrapolate from the data concerning safety, risk and ability to care that one operational definition of problematic substance use is any departure from the vigilance women are expected to exhibit, first over their pregnant bodies as vessels for foetal health, and second in relation to their primary responsibility to protect children (Bordo 2003).

Participants had little to say about either the pharmacological actions of substances or a more medicalised notion of addiction as a disease (Reinarman 2005). This is not to suggest that biomedical knowledge of the effects of substance use on foetuses and women would necessarily lend itself to a more incontrovertible conceptualisation of problematic substance use, but it is interesting that framing problematic substance use in pharmacological terms or as a biomedical disease with identifiable physical health outcomes and symptoms was often entirely absent from, or only vaguely referenced, in professional accounts of substance use. The following quote from one of the participants indicates how the pharmacological effects of drugs were given meaning via notions of maternal responsibility:

I don't make that distinction that if it's prescribed it's OK. So I'm looking at all different drug use. And, if it comes to the point where it's a, if it's higher than what could be safely used in breastfeeding ... then that's problematic. If it's impairing the mom's ability to parent the baby, then that's problematic.

Some participants expressed confusion about, or specifically noted the absence of, a body of evidence that might inform a more biomedical definition of problematic substance use among pregnant and early parenting women:

So especially if they're continuing to drink, you know, that we know that that causes, you know significant issues. For a lot of other substances, I think that the research is kind of limited about, you know, how much it affects babies. And so I try not to get super-judgmental about stuff, and really work from a harm reduction perspective, of like, 'How much can you reduce your pot smoking?' (Olivia)

I think that the latest research is indicating that any alcohol or substance exposure during pregnancy can result in a problem. I mean, there are women who drink throughout their whole pregnancy and their children are not affected. Other women, before they knew they were pregnant, they drank socially, and their children are affected, so who really knows? (Tara) 
These findings echo other sociological research that addiction as a disease remains an elusive concept. Despite the search for a specific genetic basis to addiction no physiological cause for substance use has been found, resulting in a range of suggested causal mechanisms, which has been referred to as an 'embarrassment of riches' (Reinarman 2005). It is possible that the lack of reference to biomedical effects or a disease concept among participants is because maternal substance use is regarded as so deviant that even the disease concept, which is often taken up in other discourses of addiction, is not as readily utilised in the case of maternal substance use. Another possibility is that a disease concept of problematic substance use as addiction does not often come up because, in the case of pregnant women, what is problematic about substance use has little to do with notions of addiction.

Not surprisingly, given the way substance use is associated with a reduced ability to care and wide-ranging possibilities regarding its effect on foetal and infant health, abstinence was, with only a few exceptions, regarded as the only normative substance use pattern among pregnant women. As this participant explains:

The way I would see that is a pregnant woman using a substance, whether it's alcohol or tobacco or any other drug that would have adverse impact on her foetus or herself. (Joanne)

A few respondents made specific references to how social class intersected with substance use. One participant framed the socioeconomic readability of problematic substance use within a harm reduction perspective:

A safe consumption site which can just be as simple as being housed, 'coz most of us have the opportunity to go and use what we like ... But I do it at home so nobody, it's not in front of everybody. (Emily)

However, given that any substance use by women during pregnancy and early parenting is generally regarded as problematic, harm reduction is ultimately viewed as a less desirable practice than promoting abstinence, as the following excerpts indicate:

Of course, the recommendation is that women abstain from alcohol use, they abstain from any street drug use, they abstain from smoking during, during pregnancy ... but we also have clients where they've been using crack cocaine but they actually get themselves off that and they just are smoking during the pregnancy, and when you look at that in terms of harm reduction, do I consider that problematic? Well, gosh; it would be great if she wasn't smoking, but its way better than her using throughout the pregnancy. (Sarah)

Using at all is problematic and, and we would be having that conversation with them around their use and how they feel about, decreasing their use or stopping their use, and how they think they can get there. (June)

In sum, drawing on rhetorical framings that focused on ability to care and risk and safety, substance use is regarded as a problem because it interferes with women's natural and essential responsibility to not only protect but to optimise the health of the foetus and infant child. While women's own safety and wellbeing is viewed as being connected to this principle task, 
it is subordinated under a more pressing focus on the protection of the foetus and infant child. An alternative popular framing of maternal substance use focused on women's right to define the concept for themselves.

\section{Woman as arbiter of the problematic}

When asserting that women must define problematic substance use for themselves, participants tended to deploy a couple of supporting arguments. First, some participants noted that only by allowing women to define what is problematic behaviour would they be likely to feel less judged and more likely to access health and social services such as those proposed in the HWH programme. As one participant said:

I define problematic substance use [as] how each woman would define problematic substance use in their life. So my goal in having a conversation with a woman who's using is to talk about how that's going, and if that's causing any problems in her life or what the problems are in her life, and if there's any linkages to the drug use. (Lauren)

A second, similar logic was that women are not receptive to support or changes in substance use behaviour until they come to a realisation that it is a problem:

It may be that her [health service professional] has said, 'If you engage in this use, it's going to have a negative effect on your baby, which, you know, will have a negative effect on you in your life' ... I feel like a holding of a kind of tension in that because there is such a desire to protect unborn children and to have, you know, to force women into programming, to force them to stop using substances and it's just not as simple as that. (Jennifer)

This participant suggests that the focus on women defining problematic substance use for themselves is a strategic service strategy rather than a definition of problematic substance use. Respondents who held this view may not substantially differ from their colleagues in terms of their notions of the harm of substance use among pregnant and early parenting women; however, they assert that the imposition of these views is unlikely to result in successful service encounters.

\section{Discussion and conclusion}

In writing this article, we were interested in how service providers directly or indirectly involved with a new harm reduction integrated intervention - $\mathrm{HWH}$ - define problematic substance because these definitions are likely to be influential in shaping the policies and practices of healthcare provision. The research literature indicates that problematic substance use as a kind of health behaviour is poorly understood, sometimes being viewed as deviance and disease, and most often viewed as both (Aboud and Singla 2012, Reinarman 2005, Schneider 1978). There is also intense debate on how to properly conceptualise the origins of health behaviour and the conditions and manner of agency exercised as individuals perform in different social contexts (Cockerham et al. 1997, Link and Phelan 2006, Navarro 2009). This is complicated further when we consider the case of pregnancy because the pregnant body is a site where ideological wars are engaged and competing rights claims are generated (Marcellus 2004, Wright and Walker 2007).

In addition to being interested in how theoretical models of maternal substance use as health behaviour are applied by services providers, this study was practically oriented, as several of 
the authors are involved in researching HWH and are therefore interested in a knowledge exchange that will improve service delivery. Many of the participants who took part in the interview process have contributed to the development of HWH and endorse the model that health behaviour is socially determined, as well as the need to improve healthcare delivery to pregnant and parenting women affected by substance use.

Yet most participants regarded any substance use during the reproductive period as fundamentally problematic. This framing of problematic substance use is accomplished via a gendered responsibilisation of women as foetal incubators and primary caregivers of infants. Substance use is regarded as essentially harmful, particularly in the context of the reproductive female body, despite the absence of a well-developed body of scientific evidence to support this claim (Young 1994). Furthermore, while acknowledging the role of social inequities in the production of health behaviour and health outcomes, the providers we interviewed relied largely on an individualising and moralising notion of what is problematic about substance use rather than focusing, for example, on the range of socio-structural factors that contextualise substance use (Graham 2004), the fact that substance use is both a common and widely culturally endorsed activity (Room,1983) and at the individual level, an interactional accomplishment (Becker 1967).

Similarly, although matters such as the influence of social class were invoked to portray the ways in which some subgroups of women were subjected to greater surveillance and had fewer resources to draw upon to mitigate the perceived harms of substance use, there was no substantive argument put forward by participants to suggest that the harm associated with substance use was in fact a by-product of larger systems of economic disadvantage. A possible implication of this cursory analysis of class, then, is that substance use is perceived as a risk for lower class women, while leaving unexamined the assumption that substance use is a deviant, rather than normative, activity within the context of pregnancy and parenting. The framings of substance use offered by participants do little to intervene in the social condemnation and 'stigma life sentence' associated with addiction (Lloyd 2010: 46). Indeed, it might be argued that the more ostensibly women-centred framing of problematic substance use emphasised by some of the participants, while being a potentially practical service delivery strategy, has the unintended consequence of downplaying both the role of socio-structural factors on dispositions to perceive and act and the societal responsibility to provide health and social care.

These findings are not surprising when you consider that health service providers are caught between a number of competing relevant discourses informed by neoliberalisation, the Canadian public health abstinence policy for pregnant and parenting women, the harm reduction movement, the medicalisation and criminalisation of substance use, and the deeply moral constructions of the pregnant body and motherhood, especially in Indigenous women (Kline 1993, Salmon 2011). Even government documents acknowledge that the language of substance use consists of complex and high-level discourses that often have little practical meaning at the point of care delivery. Dialectics that prioritise foetal or maternal health not only permeate popular public perceptions but also Canadian public policy on reproductive health and reproductive rights. In fact, the right of the child to protection is inscribed in legislation and the providers who took part in this study were both keenly aware of the power of this legislation to define problematic substance use and their ethical and professional duties in enacting such definitions. This recognition rests uneasy with their knowledge that many marginalised women affected by substance use avoid seeking services due to fears regarding that their child might be taken by the state (Niccols et al. 2010, Suchman et al. 2006). Flavin and Paltrow (2010) express this concern by highlighting the inherent unfairness of a system that expects disadvantaged women to provide their foetuses with health care and safety that they themselves are not able to access.

Not only did participants' conceptualisations of problematic substance use reflect the structure imposed by policy and legislation, but they are also probably conditioned by the 
allocation of resources in the health and social service sector where neoliberalisation processes have resulted in the simultaneous retrenchment of universal services and increasing surveillance of at-risk populations as well as accountability models that use the criterion of cost effectiveness of care and place the responsibility on individuals for their health and wellbeing. Service providers must forge definitions of substance use in the context of their ability to practice and the daily interactions they have with service recipients. Given their often limited role in identifying and ameliorating identified risks and the limited resources made available to address the socio-structural origins of health (that is, poverty and access to education), service providers have little opportunity to enact and sustain alternative explanations of problematic substance use.

In conclusion, our qualitative evidence supports our general conclusion above - that is, that providers' constructions of maternal substance use are largely focused on individualised notions of health agency. Their constructions are a reflection of the weighty moral forces and competing public health messages they negotiate in attempts to enact an empowering model of care in the face of dominant cultural ideals of the good mother, the hybridisation of substance use as both deviance and disease and the overall retrenchment of funding for health and social services in Canada.

These findings suggest that any health intervention programme that is grounded in moral frameworks regarding mothering and prioritises foetal and infant rights will not bode well for the delivery of an empowering and nonjudgmental model of care; rather, women's rights to health will remain subordinated and they will continue to be seen as a potential threat to their child and society. As echoed through this special issue, there is an urgent need to move away from a dominant focus on health behaviour and instead capture in research the way people carry out and make sense of health-related practices.

Address for correspondence: Cecilia Benoit, University of Victoria, Sociology, PO Box 3050, Victoria,British Columbia,V8W3P5, Canada.e-mail: cbenoit@uvic.ca

\section{Acknowledgements}

This research was supported by a Canadian Institutes of Health grant, Interventions to promote health and healthy equity for pregnant and early parenting women facing substance use and other challenges, co-principal investigators, Dr Cecilia Benoit, Centre for Addictions Research of BC \& Dept of Sociology, University of Victoria, Canada and Dr. Lenora Marcellus, School of Nursing, University of Victoria, Canada. We wish to acknowledge the editorial assistance of Ms. Marie Marlo-Barski. Above all, we wish thank the women and men who participated in interviews. Without their input, this work would not have been possible.

\section{Note}

1 Pseudonyms are used to protect the identity of the participants.

\section{References}

Aboud, F.E. and Singla, D.R. (2012) Challenges to changing health behaviours in developing countries: a critical overview, Social Science \& Medicine, 75, 4, 589-94. 
Bailey, B., McCook, J., Hodge, A. and McGrady, L. (2012) Infant birth outcomes among substance using women: why quitting smoking during pregnancy is just as important as quitting illicit drug use, Maternal and Child Health Journal, 16, 2, 414-22.

Becker, H.S. (1967) History, culture and subjective experience: an exploration of the social bases of drug-induced experiences, Journal of Health and Social Behavior, 8, 3, 163-76.

Bordo, S. (2003) Unbearable Weight: Feminism, Western Culture, and the Body. Berkeley: University of California Press.

Campbell, C. and Alexander, J. (2006) Availability of services for women in outpatient substance abuse treatment: 1995-2000, Journal of Behavioral Health Services and Research, 33, 1, 1-19.

Campbell, N.D. and Ettorre, E. (2011) Gendering Addiction: the Politics of Drug Treatment in a Neurochemical World. Basingstoke and New York: Palgrave Macmillan.

Chasnoff, M.D., Landress, H.J. and Berrett, M. (1990) The prevalence of illicit-drug or alcohol use during pregnancy and discrepancies in mandatory reporting in Pinellas County, Florida, New England Journal of Medicine, 322, 1202-06.

Claus, R.E., Orwin, R.G., Kissin, W., Krupski, A., et al. (2007) Does gender-specific substance abuse treatment for women promote continuity of care?, Journal of Substance Abuse Treatment, 32, 1, 27 39.

Cockerham, W.C. (2005) Health lifestyle theory and the convergence of agency and structure, Journal of Health and Social Behavior, 46, 1, 51-67.

Cockerham, W.C., Rütten, A. and Abel, T. (1997) Conceptualizing contemporary health lifestyles, Sociological Quarterly, 38, 2, 321-42.

Flavin, J. and Paltrow, L. (2010) Pushing pregnant drug-using women: defying law, medicine, and common sense, Journal of Addictive Diseases, 29, 2, 231-44.

Graham, H. (2004) Social determinants and their unequal distribution: clarifying policy understandings, Milbank Quarterly, 82, 1, 101-24.

Greaves, L. and Poole, N. (2008) Bringing sex and gender into women's substance use treatment programs, Substance Use \& Misuse, 43, 1271-3.

Kerker, B.D., Leventhal, J.M., Schlesinger, M. and Horwitz, S.M. (2006) Racial and ethnic disparities in medical history taking: detecting substance use among low-income pregnant women, Ethnicity and Disease, 16, 1, 28-34.

Kline, M. (1993) Complicating the ideology of motherhood: child welfare law and First Nation women, Queen's Law Journal, 18, 306.

Lefebvre, L., Midmer, D., Boyd, J.A., Ordean, A., et al. (2010) Participant perception of an integrated program for substance abuse in pregnancy, Journal of Obstetric, Gynecologic, \& Neonatal Nursing, $39,1,46-52$.

Lenton, S. and Single, E. (1998) The definition of harm reduction, Drug and Alcohol Review, 17, 2, 213-20.

Lester, B.M., Andreozzi, L. and Appiah, L. (2004) Substance use during pregnancy: time for policy to catch up with research, Harm Reduction Journal, 1, 5.

Link, B. and Phelan, J. (2006) Stigma and its public health implications, The Lancet, 367, 9509, 528-29.

Lloyd, C. (2010) Sinning and Sinned Against: the Stigmatisation of Problem Drug Users. Crowborough: UK Drug Policy Commission.

Marcellus, L. (2004) Feminist ethics must inform practice: interventions with perinatal substance users, Health Care for Women International, 25, 8, 730-42.

Murphy, E. (2000) Risk, responsibility, and rhetoric in infant feeding, Journal of Contemporary Ethnography, 29, 3, 291-35.

Navarro, V. (2009) What we mean by social determinants of health, International Journal Health Services, 39, 3, 423-41.

Niccols, A., Milligan, K., Sword, W., Thabane, L., et al. (2010) Maternal mental health and integrated programs for mothers with substance abuse issues, Psychology of Addictive Behaviors, 24, 3, 466-74.

Poole, N. and Isaac, B. (2001) Apprehensions: Barriers to Treatment for Substance-Using Mothers. Vancouver: British Columbia Centre of Excellence for Women's Health. 
Reinarman, C. (2005) Addiction as accomplishment: the discursive construction of disease, Addiction Research \& Theory, 13, 4, 307-20.

Roberts, S. and Pies, C. (2011) Complex calculations: how drug use during pregnancy becomes a barrier to prenatal care, Maternal and Child Health Journal, 15, 3, 333-41.

Room, R. (1983) Sociology and the disease concept of alcoholism. In Cappell, H. (ed) Research Advances in Alcohol and Drug Problems. New York: Plenum Press.

Rutman, D., Callahan, M., Lundquist, A., Jackson, S., et al. (2000) Substance Use and Pregnancy: Conceiving Women in the Policy Making Process. Ottawa: Status of Women Canada.

Salmon, A. (2011) Aboriginal mothering, FASD prevention and the contestations of neoliberal citizenship, Critical Public Health, 21, 2, 165-78.

Schempf, A. and Strobino, D. (2008) Illicit drug use and adverse birth outcomes: is it drugs or context?, Journal of Urban Health, 85, 3, 858-73.

Schneider, J.W. (1978) Deviant drinking as disease: alcoholism as a social accomplishment, Social Problems, 25, 4, 361-72.

Suchman, N.E., McMahon, T.J., Zhang, H., Mayes, L.C., et al. (2006) Substance-abusing mothers and disruptions in child custody: an attachment perspective, Journal of Substance Abuse Treatment, 30, 3, 197-204.

Wright, A. and Walker, J. (2007) Management of women who use drugs during pregnancy, Seminars in Fetal and Neonatal Medicine, 12, 2, 114-18.

Young, I.M. (1994) Punishment, treatment, empowerment: three approaches to policy for pregnant addicts, Feminist Studies, 20, 1, 33-57.

Zadoroznyj, M. (2006) Surveillance, support and risk in the postnatal period, Health Sociology Review, $15,4,353-63$. 